\title{
A novel form of allosteric regulation in an ancient enzyme: mapping GTP's effect on ribonucleotide reductase with SAXS and crystallography
}

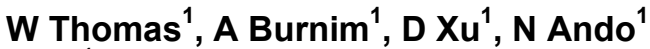 \\ ${ }^{1}$ Cornell University, Ithaca, NY \\ wct44@cornell.edu
}

Enzyme regulation is crucial to proper function, and the mechanisms that dictate this regulation often require allosteric transitions involving dynamic conformational change. A paradigm of complex regulation is the ribonucleotide reductase (RNR) family of enzymes, which uses a conserved, radical-based mechanism to catalyze the de novo conversion of ribonucleotides to deoxyribonucleotides. In previous work, we elucidated how the RNR of Bacillus subtilis maintains DNA metabolic homeostasis via an unprecedented regulatory mechanism in which active tetrameric complexes interconvert with inhibited filaments. Further work has since uncovered that $\mathrm{B}$. subtilis has evolved yet another "tuning dial" that may be linked to the organism's stress response. SAXS nucleotide titrations and chromatography-coupled SAXS experiments were used to show that the nucleotide GTP reverses RNR inhibition by breaking down inhibited filaments. Crystal structures in turn reveal a novel GTP-binding site and further suggest the mechanism of activation. This new GTP-binding site represents the surprising genesis of not just a new allosteric site but a new allosteric activator among all RNRs, and in doing so provides an exemplar of how evolutionary pressure can rapidly create novel allosteric properties.

Acta Cryst. (2020). A76, a17 